Voix et Images

\title{
La France et nous après la Seconde Guerre mondiale. Analyse d'une crise
}

\section{Robert Dion}

Volume 13, numéro 2 (38), hiver 1988

Le propre du corps Roger Des Roches

URI : https://id.erudit.org/iderudit/200711ar

DOI : https://doi.org/10.7202/200711ar

Aller au sommaire du numéro

Éditeur(s)

Université du Québec à Montréal

ISSN

0318-9201 (imprimé)

1705-933X (numérique)

Découvrir la revue

Citer cet article

Dion, R. (1988). La France et nous après la Seconde Guerre mondiale. Analyse d'une crise. Voix et Images, 13(2), 292-303. https://doi.org/10.7202/200711ar d'utilisation que vous pouvez consulter en ligne.

https://apropos.erudit.org/fr/usagers/politique-dutilisation/ 


\title{
La France et nous après la Seconde Guerre mondiale. Analyse d'une crise
}

\author{
par Robert Dion, Université Laval
}

\begin{abstract}
Aux écrivains et aux artistes canadiens qui puisent dans la jeunesse de leur pays la force de créer des auvres vivantes, vigoureuses et neuves, en témoignage d'une foi commune en l'avenir.

Robert Charbonneau, la France et nous (dédicace)
\end{abstract}

Au même titre que les livres, les événements de la vie littéraire peuvent marquer l'évolution d'une littérature. La littérature est en effet déterminée par les «spasmes» du monde littéraire. Elle n'est pas constituée seulement d'un ensemble plus ou moins grand de livres plus ou moins bons; elle est un réseau structuré (et hiérarchisé) de producteurs, d'éditeurs, de diffuseurs et de critiques, qui agissent et inter-agissent, créant ainsi la dynamique interne du champ littéraire. Ce dernier, au reste, n'est que relativement autonome: lié à la sphère économique, il est composé d'agents qui, en plus de chercher à imposer durablement certaines valeurs littéraires, s'affrontent afin de défendre des intérêts particuliers.

Non seulement les contours du champ sont déterminés par sa dynamique interne, mais ils le sont aussi par une dynamique externe: le champ s'oppose à d'autres champs, littéraires ou non. C'est ainsi que les champs littéraires distincts que constituent les littératures nationales peuvent s'affronter. L'histoire littéraire du Québec offre un cas exemplaire de ce type d'affrontement: la querelle de la France et nous 1 . La polémique eut lieu peu après la guerre, entre des intellectuels français (Aragon, Jean Cassou, André Billy, etc.) et québécois (Robert Charbonneau et Berthelot Brunet surtout), sur des questions idéologiques, littéraires et économiques. Exemple parfait de l'affrontement des littératures nationales concurrentes (de champs concurrents), cette querelle est, on le verra, l'un des événements majeurs du processus d'autonomisation de la littérature québécoise: d'où la pertinence de s'y attarder.

L'étude projetée ici est d'inspiration socio-historique: il s'agit en premier lieu de retracer les principaux moments de cette querelle franco-québécoise; aussi de

1 Robert Charbonneau, la France et nous. Journal d'une querelle, Montréal, Éditions de l'Arbre, 1947. Publication en recueil des articles écrits par Charbonneau au sujet (ou autour) de la querelle. 
décrire, en termes sociologiques, les enjeux de cet affrontement - ce qui revient à faire apparaitre l'imbrication des crises idéologique, littéraire et économique.

\section{La crise idéologique}

La «querelle» commence en février 1946 par la publication dans les Lettres françaises d'un article anonyme accusant les éditeurs canadiens de publier les ouvrages d'auteurs notoirement pétainistes et fascistes. La situation en France pendant l'Occupation avait amené les éditeurs canadiens à réimprimer les classiques et les manuels scolaires français, puis à publier les auteurs contemporains (collaborateurs ou non) ${ }^{2}$. Charles Maurras, Léon Daudet, Maurice Barrès et Pierre Drieu La Rochelle - pour ne nommer que ceux-là - figuraient alors dans les catalogues des principales maisons d'édition québécoises. Et l'on continuait, en cet immédiat après-guerre, à publier des livres de ces auteurs sans tenir compte de l'épuration qui avait cours en France sous l'égide du Comité national des écrivains (le C.N.E.).

En avril 1946, la querelle est engagée: Robert Charbonneau, directeur de la Nouvelle Relève et des éditions de l'Arbre, publie dans sa revue un article intitulé «Pourquoi ces querelles», où il se défend ainsi:

Ceux qui ont admiré avant la guerre un Maurras, un Bainville, un Massis, etc., ne l'ont pas fait parce qu'ils rêvaient d'une restauration monarchique dans un pays qui n'est pas le nôtre, mais parce qu'il se trouvait que ces écrivains étaient d'éminents représentants de la pensée française. Qu'on le nie aujourd'hui ne change rien. Le fait que Mauras [sic] et les autres ont collaboré ne change rien à ces æuvres publiées avant $1940^{3}$.

Il poursuit en affirmant n'aimer aucun de ces écrivains (sauf Daudet) et enchaîne sur une profession de foi gaulliste.

La polémique à propos de Maurras est significative, car elle révèle un malentendu fondamental. Si les écrivains du C.N.E. ne valorisent pas l'œuvre de Maurras, c'est que leur figure de classement est politique (collaborateur vs résistant); si les écrivains canadiens, en revanche, valorisent l'œuvre de cet auteur,

2 La Loi des mesures de guerre (votée en 1914) permettait aux éditeurs canadiens de réimprimer tout ouvrage littéraire passé sous la juridiction de l'ennemi. On ne mettait sous séquestre qu'un versement de dix pour cent des droits, destiné aux ayants droit français. Ce qui permit aux éditeurs québécois, suite à la défaite française de 1940, de reproduire les ouvrages français qui n'étaient plus disponibles sur les marchés. Voir à ce sujet l'article de Jacques Michon, «les Revues d'avant-garde au Québec de 1940 à 1979m, Trajectoires: Littératures et institutions au Québec et en Belgique francophone, Montréal/Bruxelles, PUM/Labor, 1985, p. 124, note 9.5 .

3 Charbonneau, op. cit., p. 19. 
c'est que leur clivage est religieux (catholique vs athée) ${ }^{4}$. Ce changement de classème explique le malentendu entre Français et Québécois au sujet des écrivains collaborateurs: ils n'interprètent pas les faits à partir de la même base de classement. D'ailleurs, faute d'une compréhension minimale, le «cas Maurras» ne constituera pas longtemps le centre de la querelle.

Pourtant, la crise idéologique est d'importance. Elle traduit une fois de plus l'écart qui, depuis la conquête anglaise, s'est peu à peu creusé entre le Canada et la France. Cet écart tient notamment à des divergences au point de vue religieux. Restée profondément catholique, la société québécoise se défie de la France, républicaine et laïque, dont l'exemple semble pernicieux. Cependant, elle garde les yeux tournés vers ce pays, qu'elle continue de considérer comme la mère patrie. La querelle naît de l'ambivalence des sentiments du Québec envers la France. On reproche à la France, pêle-mêle, son repli sur soi, son détachement des valeurs spirituelles, son manque de gratitude pour ses défenseurs 5 . On lui reproche plus précisément de n'avoir pas soutenu le travail des éditeurs canadiens:

Notre rôle terminé, écrit Charbonneau, on nous a reproché ce que nous avions fait, on n'a pas dit un mot dans les journaux français des livres que nous avions publiés, on nous reproche aujourd'hui dans les Lettres françaises de publier un livre qui déplaît à des fanatiques ${ }^{6}$.

L'accusation de fanatisme, au demeurant, est significative: elle met en lumière le caractère politique que prend la querelle du côté français. Car le «maurrassisme impénitent» - pour reprendre le titre d'un article de Jean Cassou ${ }^{7}$ —, n'est pas qu'un «travers» de l'esprit; cette adhésion à l'œuvre de Maurras s'avère plutôt, selon Cassou, un outrage à la France comme nation (comme entité politique): c'est ce qu'il souligne lorsqu'il reproche à Charbonneau son indulgence pour un ennemi de la France.

De part et d'autre de l'Atlantique, la crise idéologique donne lieu à plusieurs articles, publiés dans la Nouvelle Relève et dans les Lettres françaises. Au début de 1947, Louis Aragon fait paraître coup sur coup, dans les Lettres françaises, deux articles intitulés «Crise de l'esprit critique au Canada» et «Du courage transatlantique» 8 .

Le premier article constitue un amalgame assez complexe d'éléments ressor-

4 Dans sa réponse à Stanislas Fumet, Charbonneau précise en effet: «Mais, à la Nouvelle Relève, où n'en déplaise à $M$. Fumet, nous ne sommes pas des fascistes, même si nous sommes restés catholiques [...]». Charbonneau, ibid., p. 55.

$5 \quad$ Ibid., p. 16, 17 et 20.

6 Ibid., p. 20. Le livre incriminé est Compagnons du spirituel, de Gérard de Catalogne (Montréal, Éditions de l'Arbre, 1943).

7 Jean Cassou, «Maurrassisme impénitent», les Lettres françaises, 21 juin 1946.

8 Articles parus respectivement le 17 janvier 1947 et le 7 février 1947. 
tissant aux trois volets de la crise. Écrit en réponse à un article de Robert Charbonneau, «Crise de la littérature canadienne?»9, le texte d'Aragon traite plus ou moins directement de questions idéologiques, littéraires et économiques. Les questions idéologiques et littéraires y apparaissent étroitement mêlées. Ainsi, Aragon écrit, au sujet de l'éloge prodigué par Berthelot Brunet à l'Histoire de la littérature française de Kleber Haedens, histoire très favorable aux écrivains fascistes ou fascisants:

Je suis sûr que nos amis canadiens me comprendront si je dis qu'en face de ce dénigrement systématique de la littérature française qui va de pair avec les éloges prodigués à de tels partis pris, nous entendons ici conserver nos partis pris français, les mêmes qui valaient pendant l'occupation comme en 1946, et qui ne peuvent être que mode que pour ceux-là qui ne savent pas plus ce que signifie courage ou franchise que mode $[. .$.$] .$

En s'opposant dans le même article à Brunet et à Charbonneau, Aragon s'inscrit en faux contre deux attitudes: il met en doute, d'une part, la croyance en la soidisant «neutralité idéologique» des choix littéraires, et s'élève, d'autre part, contre la reconduction du pré-construit stipulant le déclin de la littérature française contemporaine. Il semble en effet évident pour Charbonneau (et Brunet) que les lettres françaises connaissent un déclin: cet état de fait n'est plus (de leur point de vue) à démontrer. La cause est entendue: toute la littérature française contemporaine, affirme Charbonneau, ne vaut pas le Zéro et l'infini de Kœstler. L'on observe ici, une fois encore, l'effet des différences classématiques qui fondent les divergences d'opinion, c'est-à-dire, pour reprendre les termes de Claude Lafarge, l'effet des différences entre les catégories de perception qui, en quelque sorte, constituent esthétiquement les actes de langage 10 .

Ainsi, dans cet article, Aragon lie ouvertement la question idéologique (publication des écrivains fascistes) et la question littéraire (rejet de la littérature française «qui se fait»), ce qui - on le verra - était, du côté canadien, lié depuis longtemps. Dans le deuxième article, il fait la synthèse des questions traitées dans le texte précédent; il y reprend même les classements opérés par les deux partis:
MM. Brunet et Charbonneau ont oublié de dire que j'étais un espion atomique. Ils disent, par exemple: ne confondons pas la littérature et la politique... Comme je les comprends! J'ajouterais simplement: ne con- fondons pas la trahison et la littérature.

Les valeurs sont clairement définies: la littérature est politique, l'apolitisme est trahison. En s'en prenant à l'apolitisme đéclaré des Canadiens, Aragon signale une impossibilité: la neutralité. Sur ces questions, il est intraitable. En con-

9 Charbonneau, op. cit., p. 29-35.

10 Claude Lafarge, la Valeur littéraire, Paris, Fayard, 1983, p. 14. 
séquence, il s'aliénera une grande partie des écrivains québécois qui contesteront et ses idées politiques et sa production littéraire.

\section{La crise littéraire}

Au début de la querelle, en mars 1946, Robert Charbonneau publiait un article sur «les Livres français» 11 , où il liait de façon étroite les crises idéologique et littéraire en France:

Et voici qu'après cinq ans de séparation, on nous annonce des livres de France. Que nous apporte la France? Elle nous apporte des signes d'un peuple divisé, replié sur lui-même, d'une littérature qui ne continue pas, qui n'innove pas, mais qui se recommence [...].

Ces romans, ces essais, ces récits ne sont pas ce que le public attendait d'un peuple qui, pendant cinq ans, a donné un exemple de résistance acharnée [...].

Cet état de chose indique que la crise que traverse la France n'est pas seulement une crise politique, économique ou physique, mais aussi une crise spirituelle 12 .

Selon l'auteur, la «crise» de la production littéraire française découle directement de la crise spirituelle. Les valeurs morales solides, les consensus sociaux, les régimes politiques stables apparaissent comme les conditions nécessaires et suffisantes de la «qualité» et du «rayonnement» d'une littérature. Lorsque ces conditions ne sont pas réunies, l'on assiste, comme dans l'immédiat après-guerre français, à un relatif effondrement de la culture. Une telle perception de la littérature révèle un ensemble de valeurs «pré-construites», classées selon des critères implicites; il semble que, pour Charbonneau, la littérature se range d'abord sous un classème /moral/ (primauté du spirituel), tandis que chez Aragon, la figure de référence est/idéologique/ (au sens restreint). Jamais, cependant, ces classèmes ne sont explicités. Formés ailleurs, ils investissent, déjà «tout faits», le discours; ils s'avèrent essentiellement des figures doxiques.

Par suite de l'interdiction au Québec, durant l'été de 1947, du film les Enfants du paradis, la crise franco-canadienne continue de s'envenimer: cette interdiction apparaît, du côté français, comme le résultat d'une offensive délibérée contre la culture française. François Mauriac aura à ce propos quelques mots acerbes et préconisera l'envoi massif en territoire canadien des œuvres de Delly (qui ne sauraient évidemment être jugées subversives).

Tout en souhaitant que la littérature québécoise se dégage des fins ou des commandes politiques, sociales ou historiques, Robert Charbonneau insiste sur

11 Charbonneau, op. cit., p. 16-18.

12 Ibid., p. 17. 
les données politiques favorisant l'éclosion d'une littérature nationale. Une littérature ne peut être, selon lui, que nationale. L'indépendance politique (canadienne ${ }^{13}$, en l'occurence, et non québécoise) constitue un préalable nécessaire à la naissance de grandes œuvres 14: les grandes époques des littératures nationales correspondraient aux époques de suprématie militaire ou politique des nations. Ainsi, sur la base de ces postulats, Charbonneau peut lier l'affirmation de la littérature canadienne-française à celle du Canada sur la scène internationale. L'autonomie du pays préfigure l'autonomie des lettres. Cela n'est pas sans susciter certaines ambiguittés - eu égard notamment au fait que Charbonnerau ne sait pas s'il doit accorder la primauté au spirituel, au politique ou au littéraire.

Du côté français, d'autres interlocuteurs débattent de la question littéraire. Car si Aragon enjoignait aux Québécois de faire preuve dans leurs choix littéraires d'esprit critique, il n'intervenait pas dans les affaires littéraires canadiennes et ne tentait pas de gommer la spécificité des lettres canadiennes. Cette occultation est plutôt le fait de Georges Duhamel, de Stanislas Fumet, d'André Billy (et d'un Canadien, René Garneau).

Déjà, le 4 janvier 1946, George Duhamel publiait dans le Figaro un article, "Chronique. L'Arbre et la branche», qui mettrait bientôt le feu aux poudres. Il y affirmait: Le monde canadien est une branche de l'arbre français, une branche robuste et qui semble maintenant séparée du tronc original par une épaisse muraille: une branche quand même et qui fait honneur à l'arbre, à la vitalité de l'arbre. Curieusement, le principal interlocuteur de Duhamel fut un autre Français, Étienne Gilson, qui faisait paraître dans le Monde du 6 janvier 1946, sous le titre «l'Arbre et la branche», cette réponse:

[Le Canada] se souvient d'abord d'avoir été une branche de l'arbre français, mais aussi d'en avoir été coupé, puis, laissé sur le sol, d'y avoir pris tout seul racine, d'avoir vécu sans nous, pensé sans nous, grandi sans nous [...]. Si nous sommes l'arbre, jamais arbre ne s'est moins soucié de sa branche. Qu'il s'en soucie aujourd'hui, rien de mieux, mais ce qu'il retrouve, après l'avoir si longtemps négligé, ce n'est plus une branche, c'est un arbre: un arbre de même espèce que lui, mais un autre arbre, qui est un arbre comme lui.

Robert Charbonneau fait allusion à cet échange en juin 1946, y revient en octobre-novembre 1946 et plusieurs fois par la suite. Cet article de Gilson (et cet autre article, «Depuis le XVIII e siècle, le Canada a sa littérature originale»15, qui rapporte le début de la littérature canadienne à la conquête anglaise) lui est prétexte à affirmer l'autonomie de la littérature québécoise.

13 Charbonneau n'hésite pas à se féliciter de l'existence, au Canada, de deux langues littéraires.

14 Charbonneau, op. cit., p. 21.

15 Étienne Gilson, «Depuis le XVIIIe siècle, le Canada a sa littérature originale», Une semaine dans le Monde, 26 avril 1947. 
Toute la France et nous vise à affirmer cette autonomie nouvelle, difficilement conquise: en face de la France, se pose le nous, entité homogène, tangible, qui peut s'opposer à l'autre. Et même si, comme le relève André Billy, la littérature française est antérieure à la littérature canadienne, cela n'explique pas, écrit Charbonneau, que l'arbre français ne pût donner naissance qu'à des branches, et non à une forêt ${ }^{16}$. Cela, du reste, ne signifie pas non plus que le canada dût rester éternellement, comme le veut Stanislas Fumet, un élève du maître français 17 . La querelle de la France et nous représente un grand moment pour la littérature canadienne. Elle permet de revendiquer haut et clair la spécificité d'une littérature nationale et de cristalliser certains de ses enjeux. Les aspirations autonomistes des écrivains québécois se font jour. Cette résurgence est très importante: elle l'est à ce point que la plupart des analystes ne verront que la querelle littéraire, sans chercher si celle-ci n'est pas, en profondeur, déterminée par des facteurs économiques.

Si la querelle ne signe pas nécessairement, dit-on en règle générale, l'acte de naissance d'une littérature québécoise autonome ${ }^{18}$, elle est néanmoins l'occasion d'affirmer la volonté d'indépendance des écrivains. Celle-ci se manifeste non seulement vis-à-vis des autorités religieuses et politiques du Canada, mais aussi (et surtout) par rapport aux autorités littéraires françaises. À cette fin, Charbonneau invoque les influences américaines, anglaises ou russes. En plus de revendiquer son «américanité», il revendique pour Paris, New York et, par extension, Montréal, un même statut de métropole culturelle.

Charbonneau signale trois conditions de l'autonomisation du champ littéraire, lesquelles sont désormais remplies au Québec: un milieu intellectuel favorable, des cadres matériels propices (maisons d'édition, etc.) et des techniques littéraires «originales». Toutes ces conditions définissent la circularité du champ littéraire et permettent à l'écrivain d'inscrire ses pratiques dans une aire où sont concentrées les instances de légitimation (qui échappent au contrôle des milieux non littéraires). Autrement dit, la «clôture» du champ littéraire et la spécialisation des diverses instances qui le composent permettent à l'écrivain de manœuvrer dans un monde où, semble-t-il, n'ont cours qué de pures valeurs symboliques.

Selon Charbonneau, les techniques littéraires originales proprement cana-

16 Charbonneau, op. cit., p. 57.

17 Ibid., p. 55.

18 Des traces du procès d'autonomisation de la littérature québécoise semblent en effet se lire plus tôt, vers 1920, avec la querelle des «régionalistes» et des «exotiques» (voir à ce sujet l'article de Marie-Andrée Beaudet, «Petit historique du procès d'autonomie de la sphère littéraire au Québec», Actes du premier colloque des étudiants gradués du CRELIQ, Québec, CRELIQ, 1986, p. 11-16). Nous ne sommes ni au début ni à la fin du processus d'autonomisation des lettres québécoises. En fait, ce qu'il faut noter ici, c'est la lucidité avec laquelle Robert Charbonneau décrit les voies de l'autonomisation: il le fait en des termes qui, quelques décennies plus tard, seront ceux des praticiens de l'analyse institutionnelle ou de la sociologie culturelle. 
diennes doivent provenir de plusieurs influences combinées: cela semble, de prime abord, paradoxal, mais Charbonneau souscrit à l'idée selon laquelle faire «jouer» ensemble de nombreux emprunts est susceptible de donner naissance à une certaine forme d'originalité. Celle-ci sera renforcée, sur le plan thématique, par le recours à des éléments caractéristiques du milieu canadien. De sorte que nos écrivains,

[...] s'ils se rattachent encore à des écoles françaises ou américaines, visent à se dégager de tous liens et [qu']on peut prévoir qu'il sortira de cette génération des œuvres intégralement canadiennes d'une portée universelle 19 .

Cette génération se manifeste vers 1940 , lors de l'apparition des premières maisons d'édition canadiennes. Elle est, selon Charbonneau, de formation canadienne; elle est composée d'écrivains qui se sont «trouvés eux-mêmes», par suite de longues méditations sur les cuvres étrangères 20 . Encore une fois, l'on retrouve ici l'ambiguïté foncière de Charbonneau, cette hésitation entre la revendication d'une pure «canadianité» et celle d'influences étrangères. Cette oscillation lui vaudra d'ailleurs l'attaque de René Garneau (écrivain canadien résolument non autonomiste): Pour bien marquer leur scission avec la France, ils [les jeunes écrivains canadiens] invoquent leur parenté spirituelle avec les écrivains américains. Or on croyait que c'était l'autonomie qu'ils voulaient 21 . Mais peutêtre faut-il discerner, dans l'américanophilie de Robert Charbonneau, un opportunisme commercial: les États-Unis restent, pour le livre français édité au Québec comme pour le livre canadien, un marché potentiel immense - et fort attirant.

\section{La crise économique}

En fin de compte, ces observations renvoient à la question économique. Celle-ci s'avère en effet déterminante. Ces quelques chiffres soulignent l'essor considérable qu'a connu l'édition canadienne-française entre 1940 et 1946:

Durant cette période, il s'imprime 21 millions de livres au Québec. Avant la guerre, en 1939, le Canada français ne compte que quelques éditeurs littéraires (Edouard Garand et Albert Lévesque par exemple); en 1940, peu après la défaite française, trois éditeurs déjà en place deviennent des éditeurs littéraires (Beauchemin, Granger et frères, Valiquette); quatre maisons sont fondées en 1941 (Fides, l'Arbre, Variétés et Pony); entre 1943 et 1945 enfin, huit autres nouvelles maisons sont fondées (Parizeau, Pascal, Serge, Marquis, Lumen, Mangin, B.D. Simpson et Pilon). Dans le sillage de ces nouvelles maisons d'éditions, naissent quelques nouvelles revues, dont la Nouvelle Relève (1941-1947), Regards, Amérique française (1944-1946). Par ailleurs, on fonde,

19 Charbonneau, op. cit., p. 23.

20 Ibid., p. 25.

21 Cité par Charbonneau, ibid., p. 31. 
en 1943, la Société des éditeurs canadiens du livre français, organisme qui veut promouvoir les intérêts des éditeurs.

En 1945, les éditeurs canadiens avaient publié 120 ouvrages de littérature (romans, contes, essais, poèmes), 60 ouvrages d'histoire ou de géographie, 25 ouvrages d'actualité, 75 ouvrages de philosophie ou de sociologie, environ 30 ouvrages de médecine, 200 ouvrages de littérature de jeunesse, 160 manuels et 700 rééditions d'ouvrages français; la seule maison Variétés, par exemple, publie entre 1941 et 1945 la «somme» de 358 ouvrages 22.

Cependant, dès 1945, la manne s'épuisa. Aux lendemains de la libération, les éditeurs canadiens durent déchanter. Dès la fin de la guerre en effet, le syndicat français de la librairie s'insurgea contre les privilèges accordés aux éditeurs canadiens et se plaignit des droits trop peu élevés (10\%) exigés de ces derniers. Or, ces privilèges avaient permis aux éditeurs canadiens de connaître la prospérité: la publication des livres français leur donnait une assise financière solide, de même qu'une assise symbolique leur permettant de publier (d'imposer) les auteurs canadiens et de créer un marché local. Malheureusement; peu d'éditeurs québécois réussirent, pendant la guerre à se constituer un fonds qui pût leur assurer des revenus fixes: d'où les nombreuses faillites (entre 1946 et 1949, disparaissent près de $70 \%$ des maisons d'édition littéraires, soit douze entreprises).

Georges Duhamel, qui représentait au Canada le syndicat français - c'est à ce moment qu'il énonça la métaphore de l'arbre -, chercha à trouver un compromis avec les éditeurs canadiens. Il leur proposa, comme l'évoque Aragon dans l'un de ses articles, d'acquitter en papier les droits de publication des œuvres françaises (le papier, pendant la guerre, avait manqué à la France). Cette fois encore, les Français saisirent mal les véritables enjeux de la querelle: il ne pouvait être question, pour les Canadiens, de confondre valeur économique et valeurs symboliques. Ces derniers, bien entendu, réagirent vivement. Il se trouva même un Français, Auguste Viatte, pour s'indigner des propositions françaises:

Comme si un éditeur pouvait se transformer du jour au lendemain en marchand de papier, et comme si d'ailleurs les grandes firmes angloaméricaines qui font le commerce du papier jouaient dans la diffusion de notre culture un rôle comparable à celui de l'éditeur canadien-français! Il en est résulté des polémiques regrettables, et qui ont dévié sur le terrain

22 Ces chiffres proviennent de trois sources:' l'article de Lucie Robert, «Prolégomènes à une étude sur les transformations du marché du livre au Québec (1900-1940)», l'Imprimé au Québec, aspects historiques (18e-19e siècles), Québec, IQRC, 1983, p. 225-242; le livre du Groupe de recherche sur l'édition littéraire au Québec (GRELQ), l'Édition littéraire au Québec de 1940 à 1960, Sherbrooke, Département d'études françaises, 1985 , p. 3-26; la publicité rédigée par Robert Charbonneau, «Hommage aux éditeurs canadiens», le Canada, 22 octobre 1945, p. XVIII. 
littéraire23.

Certaines maisons d'édition canadiennes purent s'entendre avec des maisons françaises et conclure des ententes particulières. Mais la majeure partie des éditeurs, on le sait, firent faillite.

Dans la France et nous, Robert Charbonneau fait évidemment allusion aux questions éditoriales et à sa propre fonction d'éditeur. Il y reprend entre autres le «Discours prononcé au congrès de la société des éditeurs canadiens» 24 , dans lequel il prétend traiter du développement de l'édition canadienne. Or, Charbonneau ne parle pas vraiment de l'édition, ni a fortiori des problèmes économiques de ce secteur d'activité, pourtant cruciaux au moment où, en 1947, il prononce ce discours. Il ne traite en fait que de l'affirmation des lettres canadiennes, dont semble tout naturellement dépendre la santé de l'édition. Tout se passe en somme comme si la crise économique n'avait pas lieu. Du reste, au plus fort de la crise, en 1948, Charbonneau publie dans le Canada un discours triomphaliste, «l'Édition canadienne a pour mission de révolutionner notre littérature» 25 , où il n'êvoque pas une seule fois la crise de l'édition; tout au plus note-t-il, en conclusion, qu'après

[...] avoir révolutionné la littérature, l'édition doit maintenant révolutionner ses méthodes et ses procédés. Il faut abandonner les méthodes désuètes de la librairie; le succès est à ce prix. Car une industrie du livre progressive [sic] et forte est essentielle à la littérature [...].

Il est significatif de comparer la position de Charbonneau (telle qu'exprimée dans la France et nous ou dans cet article) à celle de la Société des éditeurs canadiens exprimée en 1948 dans la Revue de l'Université Laval26. Contrairement à Charbonneau, la Société des éditeurs met au premier plan les difficultés économiques de l'édition canadienne et propose, pour y remédier, une série de mesures concrètes, dont la création d'une chambre de compensation qui permettrait de régler le flux des exportations de livres canadiens en France et des importations de livres français au Canada.

Charbonneau ne traite de l'édition que sous deux aspects: soit comme condition théorique du rayonnement des lettres, soit comme moyen empirique de susciter la production littéraire. Il ne perçoit jamais l'édition comme une instance qui, soumise à des pressions économiques (la concurrence par exemple), sélec-

23 Auguste Viatte, «Édition canadienne et livre français», le Monde, 20 mars 1948, repris dans le Devoir, 10 avril 1948, p. 10.

24 Charbonneau, op. cit., p. 43-47.

25 Robert Charbonneau, «l'Édition canadienne a pour mission de révolutionner notre littérature», le Canada, 20 février 1948, p. 4. Cet article est le texte intégral d'une conférence prononcée le 19 février 1948 devant la Société des éditeurs canadiens.

26 «L'importance des livres canadiens en France», Revue de l'Université Laval, vol. 2, $\mathrm{n}^{\circ} .8$ (avril 1948), p. 749-750. 
tionne et consacre des œuvres. L'édition est réduite à la tâche, noble il est vrai, de conseil littéraire. En fait, quoique tous les articles recueillis dans la France et nous aient été rédigés entre février 1946 et juin 1947, ils ne semblent guère dater - eu égard à la façon dont ils traitent de la question éditoriale - de l'une de ces deux années, mais plutôt de la très prospère période 1940-1945.

Si en apparence Charbonneau ne professe, en plus d'un nationalisme politique, qu'un nationalisme littéraire, ce dernier se double cependant d'un nationalisme économique. Par-delà les discours, toute l'action de Charbonneau, comme président de la Société des éditeurs canadiens ou comme directeur des éditions de l'Arbre, vise à créer et à maintenir un marché national indépendant et à régir les importations de livres français au Canada. Mais cette action, au même titre que les rivalités éditoriales entre la France et le Canada, est occultée. Charbonneau passe sous silence les luttes économiques «triviales», qui s'avèrent pourtant déterminantes; car, comme l'affirme Lucie Robert:

L'édition québécoise des années 1940 et 1950 est [...] prise dans un étau. D'une part, les librairies locales sont dépendantes du marché institutionnel [scolaire]; d'autre part, les succursales de librairies européennes se consacrent à l'importation et à l'écoulement de leur stock ${ }^{27}$.

Dès cette époque en effet, les éditeurs français tentent d'envahir le marché canadien. En 1950, la Librairie Hachette fait un geste décisif en rachetant les Éditions Aristide Pony. La même année Flammarion ouvre un comptoir à Montréal.

Rappelons ici que les enjeux économiques de la querelle sont très importants. S'il s'agit, pour les éditeurs canadiens (résolus à perdre le marché mondial du livre français), de maintenir le marché local qu'ils estiment avoir eux-mêmes créé, il faut voir que, du côté français, les enjeux ne sont guère négligeables. Certes, la conquête du marché canadien n'est pas essentielle: bien moins, en tout cas, que la reconquête du marché intérieur français, ou des marchés américain, européen et latino-américain. Toutefois, la question du papier reste primordiale. Pour les éditeurs français, la reconquête des marchés passe par la fabrication des livres au meilleur prix; d'où l'importance de pouvoir s'approvisionner facilement en papier, et à bon prix. De fait, la question du papier est si importante qu'elle va donner lieu à des alliances inattendues, notamment celle d'Aragon et de Georges Duhamel.

Ainsi, la question économique ne commence à apparaître vraiment qu'en 1948 , quand la querelle est presque terminée. Ce n'est en outre qu'en 1951 que le rapport Massey sur «l'avancement des arts, des lettres et des sciences» la met véritablement à l'ordre du jour. Pourtant, Auguste Viatte, dans l'article que nous avons déjà cité, insistait nommément sur l'antériorité de la crise économique sur les crises idéologique et littéraire. Car c'est, rappelons-le, dès 1946 que Georges Duhamel est envoyé au Canada pour conclure de nouvelles ententes éditoriales.

27 Robert, op. cit., p. 237. 
Le problème de l'édition se répercute directement dans la sphère littéraire. La littérature canadienne, qui s'était développée de pair avec l'édition, sort de la guerre anémiée. De toute évidence, ce qui est en jeu dans le problème de l'édition, c'est l'autonomie du champ littéraire québécois, qui dépend au premier chef de la maîtrise des moyens de production et de diffusion des œuvres littéraires et de la survie d'un marché littéraire québécois indépendant.

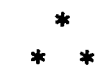

Il semble en définitive que les polémiques littéraires et idéologiques furent, avant tout, une guerre économique entre une «puissance» éditoriale majeure (la France) et une infrastructure éditoriale naissante (les éditeurs canadiens-français). En fait, ces trois crises (idéologique, littéraire, économique) se superposent, constituant les avatars d'une même crise - au premier chef économique. Par un jeu de sublimation (car il n'est évidemment pas question, en littérature, de s'intéresser à l'argent), le vide économique créé dans l'édition québécoise par la reprise de l'activité éditoriale française s'est transmué en querelles éthiques et esthétiques. Autrement dit, les crises idéologique et littéraire constituèrent à proprement parler des dérivatifs, dont la fonction fut de détourner l'attention des problèmes économiques. Car en déplaçant la querelle sur le terrain idéologique ou littéraire, Charbonneau put occulter la question quasi insoluble (et extra-littéraire) du commerce franco-canadien du livre et ramener au premier plan la «querelle des valeurs». Celle-ci, qui se joua sur tous les plans (revendications des influences, réception critique des œuvres, etc.), permit à Charbonnneau de ranimer encore une fois un débat fondamental mais qui devenait de plus en plus oiseux, au fur et à mesure que la littérature québécoise se distinguait de la littérature française et que les valeurs des deux peuples devenaient aussi indiscutables que leurs coutumes. Or, c'est précisément parce qu'elle permet d'occulter d'autres questions que la querelle des valeurs est si importante: tout au long de l'histoire littéraire québécoise, elle servira à masquer des problèmes économiques, idéologiques / linguistiques ou littéraires.

Disons pour terminer que les problèmes de l'édition canadienne vont peu à peu cesser de se poser avec une telle acuité. À la fin des années quarante s'amorce une renaissance de l'édition, notamment en poésie, avec la fondation des éditions Erta (1949), des éditions de l'Hexagone (1953), etc. Ces nouveaux éditeurs vont imposer une autre littérature, liée à la cause d'un nationalisme de type nouveau, non plus canadien, mais québécois. 